\section{Case Reports in Ophthalmology}

Case Rep Ophthalmol 2020;11:276-281

DOI: 10.1159/000508424

Published online: July 3, 2020
(C) 2020 The Author(s)

Published by S. Karger AG, Basel www.karger.com/cop

This article is licensed under the Creative Commons Attribution-NonCommercial 4.0 International License (CC BY-NC) (http://www.karger.com/Services/OpenAccessLicense). Usage and distribution for commercial purposes requires written permission.

\title{
A Rare Case of Unilateral Progressive Cataract in a Young Patient Receiving Topiramate
}

\author{
Tanuja Kate Rajiv Choudhary Jyoti Singhai Navita Pathak
}

Rajas Eye and Retina Research Centre, Indore, India

\section{Keywords}

Topiramate · Glaukomflecken · Progression of cataract · Ciliochoroidal effusion · Secondary angle closure

\begin{abstract}
This case report presents an instance of unilateral cataract formation and its rapid progression following topiramate-induced bilateral acute angle closure. An 18-year-old female diagnosed with acute angle closure in both eyes had started treatment on the previous day at another healthcare facility. The patient presented with complaints of pain, sudden diminution of vision, excessive watering, and photophobia (both eyes) and reported the use of topiramate for headache for 10 days. There was no past history of decreased vision, trauma, uveitis, or use of steroids. Topiramate-induced bilateral secondary angle closure attack was the presumptive diagnosis. Topiramate use was stopped, and antiglaucoma drugs, topical cycloplegic, and topical steroids were started. On 1-day follow-up, clearer cornea and peripheral anterior capsular lenticular opacity of the right eye were observed. Gonioscopy showed closed angles. Anterior segment optical coherence tomography showed forward movement of the iris-lens diaphragm and closed angles. B-scan showed ciliochoroidal effusion in the right eye and normal left eye. At 2-month follow-up, formed anterior chamber and posterior subcapsular cataract in the right eye were seen. There were no lenticular changes in the left eye. Definite progression of cataract from day-1 to 2-month follow-up was seen in the right eye. To our knowledge, this is the first report of the rapid progression of cataract following topiramate-induced secondary angle closure in a young patient warranting surgical intervention.




\section{Case Reports in Ophthalmology}

\section{Introduction}

Topiramate is an oral sulfamate-substituted monosaccharide used in the treatment of epilepsy, migraine, and neuropathic pain [1]. Its use has been associated with secondary angle closure, which can mimic acute primary angle closure [1]. The suspicion for medicationinduced angle closure is higher for bilateral presentation. This report presents a case of topiramate-induced bilateral secondary angle closure, with development and rapid progression of the cataract in one of the eyes, warranting surgical intervention.

\section{Case Report}

An 18-year-old female had been diagnosed with bilateral acute angle closure at a different healthcare facility. She had been prescribed systemic antiglaucoma drugs and topical betablocker, pilocarpine, and prostaglandin analogue as treatment. The patient presented with complaints of pain, sudden diminution of vision, excessive watering, and photophobia (both eyes). In the course of history gathering, the patient reported the use of topiramate $25 \mathrm{mg}$ twice a day for headache for 10 days. There was no history of trauma or symptoms suggestive of uveitis. The patient did not report use of any drug other than topiramate. She had a history of headache and there was no family history of glaucoma. Her visual acuity was right eye (OD) counting fingers 2 feet and left eye (OS) counting fingers 4 feet. The eyelids were swollen. Slit lamp examination revealed mild chemosis, ciliary flush, diffuse stromal haze (OD > OS), microcystic corneal edema OS, and very shallow peripheral anterior chamber with areas of iridocorneal touch (OD > OS). The pupil was semidilated and sluggishly reacting without rapid afferent pupillary defect. Intraocular pressure was OD $40 \mathrm{~mm} \mathrm{Hg}$ and OS $32 \mathrm{~mm} \mathrm{Hg}$.

A presumptive diagnosis of topiramate-induced bilateral secondary acute angle closure attack was made. Topiramate use was stopped. Systemic and topical antiglaucoma drugs (intravenous mannitol $300 \mathrm{~mL}$ stat, acetazolamide $250 \mathrm{mg}$ twice daily for a day, topical fixed drug combination of dorzolamide and timolol thrice daily, brimonidine $0.1 \%$ twice daily), topical cycloplegic (homatropine 1\% twice daily), and a steroid (low-dose dexamethasone four times a day) were started.

On 1-day follow-up, the cornea had become clearer in both eyes (Fig. 1a, b) and the right eye showed peripheral anterior capsular lenticular opacity (Fig. 1a). There was no aqueous flair and the vitreous was clear. Fundus examination showed normal optic disc, retina, and macula. Intraocular pressure had decreased to OD $12 \mathrm{~mm} \mathrm{Hg}$ and OS $10 \mathrm{~mm} \mathrm{Hg}$. Gonioscopy showed closed angles. Anterior segment optical coherence tomography (ASOCT) (Fig. 2a, b) showed forward movement of the iris-lens diaphragm with closed angles. B-scan of right eye (Fig. 3a) showed ciliochoroidal effusion, while B-scan of the left eye (Fig. 3b) was normal. At 1-week follow-up, ASOCT showed the iris-lens diaphragm to have moved back with open angle (Fig. 2c, d). One week after presentation, all antiglaucoma and cycloplegic drugs were stopped and the patient was continued on low-dose steroid for another week. At 2-month follow-up, the right eye showed formed anterior chamber and increased density of cataract, while the left eye did not show any lenticular changes (Fig. 1c, d). Thus, in right eye, there was a definite progression of cataract from 1 day to 2 months (Fig. 1e, f), and hence cataract surgery was advised. 


\section{Case Reports in Ophthalmology}

\section{Discussion}

Topiramate-induced bilateral angle closure glaucoma is well described [2-6]. Mahendradas et al. [7] recently showed topiramate-induced angle closure glaucoma with severe panuveitis, which resolved with systemic and topical steroids and cessation of topiramate. In this case, the cataract developed owing to severe uveitis. In the present case report, acute angle closure, forward movement of the iris-lens diaphragm, and iridocorneal touch (OD > OS) resulted in glaukomflecken in the right eye as against left eye; previously published literature has also demonstrated glaukomflecken in acute angle closure [8]. Also, there were no signs of uveitis and still posterior subcapsular cataract developed unilaterally and progressed rapidly. Therefore, this case report suggests that in topiramate-induced bilateral secondary angle closure, there is a possibility of development and rapid progression of posterior subcapsular cataract warranting surgical intervention, and hence one must be vigilant in following up these cases.

However, there are some limitations to this study. Firstly, there was no objective measurement of anterior chamber depth to reveal any significant asymmetric chamber depth. Secondly, the investigations were not focused on all bilateral assessments at the first visit, and therefore retroillumination figures of this visit are not available. In our study, the formation of posterior subcapsular cataract after angle closure is at best presumptive and we cannot establish or deny the cause-effect relationship between topiramate-induced angle closure and progression of unilateral cataract with the current findings. The cataract formation in the right eye could be presumably explained by ciliochoroidal effusion, which was evident only in the right eye. Development of cataract in the presence of ciliochoroidal effusion has also been shown in earlier reports $[9,10]$.

To our knowledge, this is the first report of cataract development and rapid progression without any associated inflammation following topiramate-induced secondary angle closure in a young patient. Therefore, if angle closure presents, especially bilaterally, a detailed history and review of medications is mandatory, and these patients must be followed up on a regular basis so as to see any appearance and/or progression of cataract.

\section{Acknowledgements}

The authors thank Dr. Nilesh Kumar Jain (Knowledge Shapers, Indore) for medical writing and editorial assistance.

\section{Statement of Ethics}

This study was conducted in accordance with the ethical requirements of the Declaration of Helsinki; prior written informed consent for publication of her case (including images) was obtained from the patient.

\section{Conflict of Interest Statement}

The authors declare that there is no conflict of interest in relation to the current publication. 


\section{Case Reports in Ophthalmology}

\section{Funding Sources}

No financial support was received for this study.

\section{Author Contributions}

T. Kate and R. Choudhary analyzed and interpreted the patient data. All authors reviewed and approved the final manuscript.

\section{References}

1 De Oliveira BMR, Ferrari PV, Herrerias BT, Hirai FE, Gracitelli CPB. The use of topiramate for weight loss causing acute glaucoma: a case report and literature review. Med Hypothesis Discov Innov Ophthalmol. Summer 2019;8(2):116-20.

2 Lan YW, Hsieh JW. Bilateral acute angle closure glaucoma and myopic shift by topiramate-induced ciliochoroidal effusion: case report and literature review. Int Ophthalmol. 2018 Dec;38(6):2639-48.

3 Caglar C, Yasar T, Ceyhan D. Topiramate induced bilateral angle-closure glaucoma: low dosage in a short time. J Ocul Pharmacol Ther. 2012 Apr;28(2):205-7.

4 Joshi AK, Pathak AH, Patwardhan SD, Kulkarni AN. A rare case of topiramate induced secondary acute angle closure glaucoma. J Clin Diagn Res. 2017 Jun;11(6):ND01-3.

5 Behl S, Fasahtay A. Topiramate-induced bilateral angle closure glaucoma and myopic shift. Neurol India. 2016 Sep-Oct;64(5):1040-2.

6 Kulkarni C, Chaudhuri UR, Jagathesan A. Bilateral acute angle-closure glaucoma following treatment with topiramate for headache. Neurol Ther. 2013 Mar;2(1-2):57-62.

7 Mahendradas P, Parab S, Sasikumar R, Kawali A, Shetty BK. Topiramate-induced acute angle closure with severe panuveitis: A challenging case report. Indian J Ophthalmol. 2018 Sep;66(9):1342-4.

8 Lowe RF. Primary acute angle-closure glaucoma damage to cornea and lens. Br J Ophthalmol. 1965 Sep; 49(9):460-5.

9 Stelmach MZ, O’Day J, Ryan H. Uveal effusion syndrome. Aust N Z J Ophthalmol. 1994 May;22(2):139-43.

10 Reddy AC, Salim S. Diagnosis and management of choroidal effusions. www.aao.org/eyenet/article/ choroidal-effusions [accessed April 26, 2020]. 

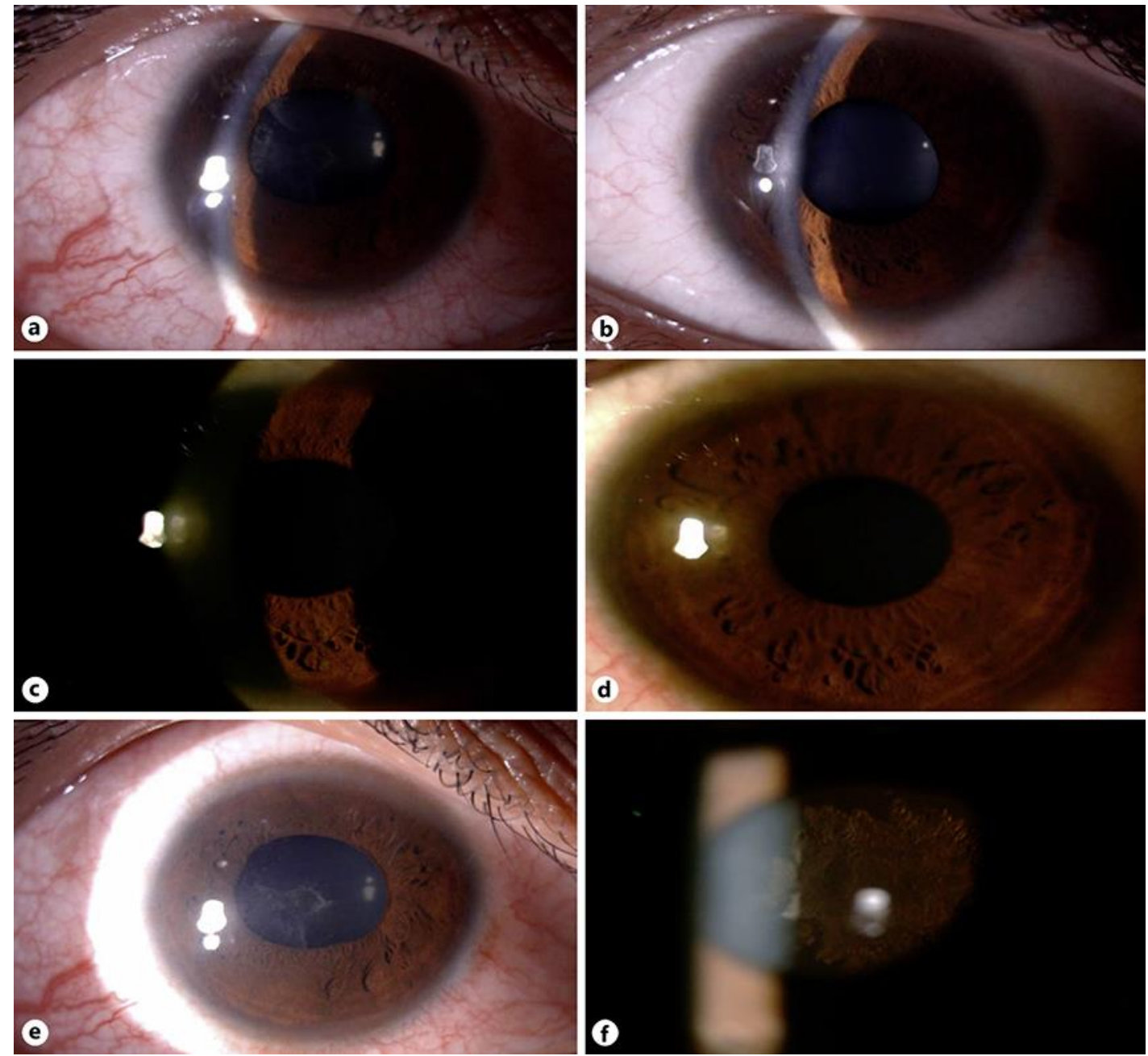

Fig. 1. Slit lamp examination. a, b Slit lamp findings of the right (a) and left (b) eyes on 1-day follow-up. The right eye showed peripheral anterior capsular lenticular opacity, while in the left eye the lens was clear. c, $\mathbf{d}$ Slit lamp findings of the left eye at 2-month follow-up. e, f Slit lamp examination findings of the right eye on 1-day (e) and 2-month (f) follow-up. e Localized anterior capsular lenticular opacity in the right eye on 1-day follow-up which increased in density in due course of time. $f$ At 2-month follow-up there was increased density of cataract. It is evident that at 2-month follow-up, the right eye showed formed anterior chamber. The left eye did not show any lenticular changes. Thus, in the right eye, there was definite progression of cataract from 1-day to 2-month follow-up. 
Case Reports in Ophthalmology
Case Rep Ophthalmol 2020;11:276-281

DOI: 10.1159/000508424

(c) 2020 The Author(s). Published by S. Karger AG, Basel www.karger.com/cop

Kate et al.: Unilateral Progressive Cataract in a Young Patient Receiving Topiramate
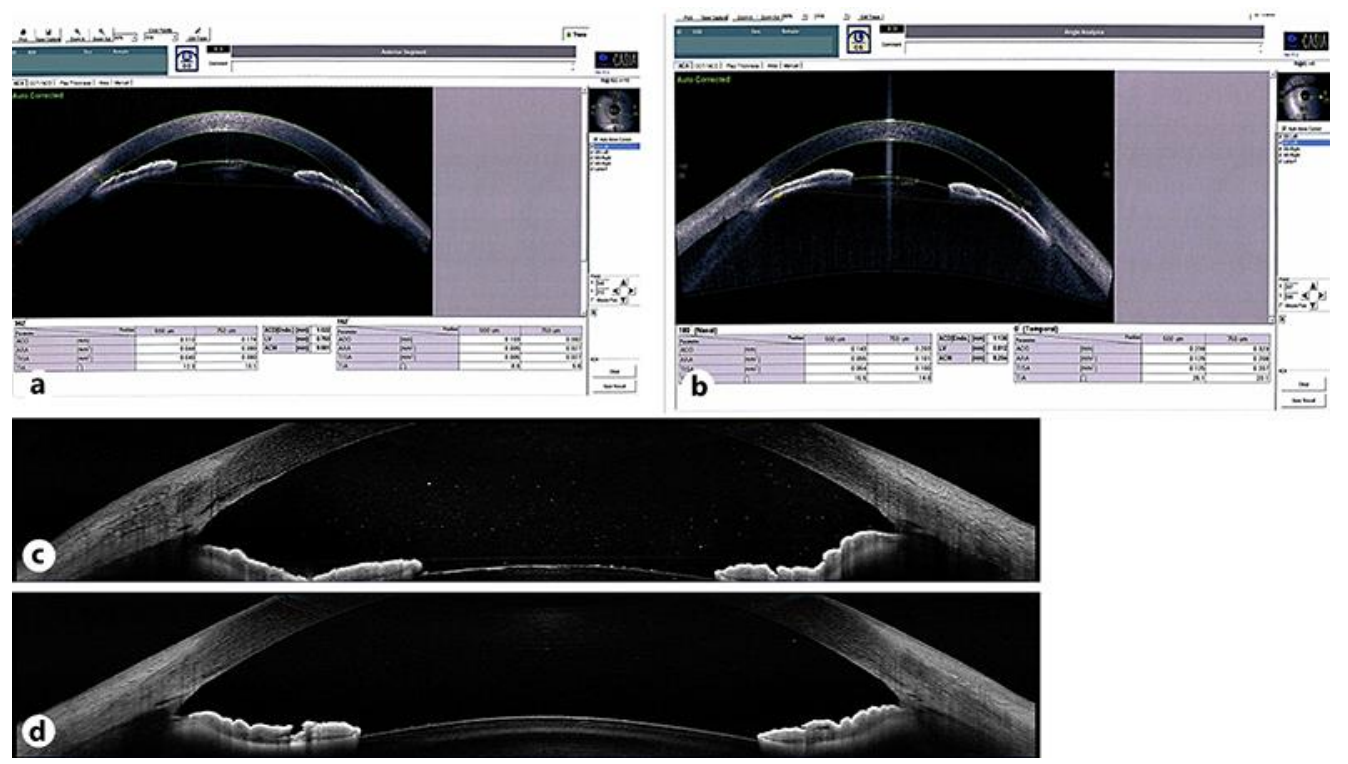

Fig. 2. ASOCT findings. a, b ASOCT findings of right (a) and left (b) eye at presentation. ASOCT showed forward movement of the iris-lens diaphragm and closed angles in both eyes. c, d ASOCT findings of the right (c) and left (d) eye at 1-week follow-up. At 1-week follow-up in both the eyes ASOCT showed the irislens diaphragm to have moved back and the angle was seen to be open. ASOCT, anterior segment optical coherence tomography.
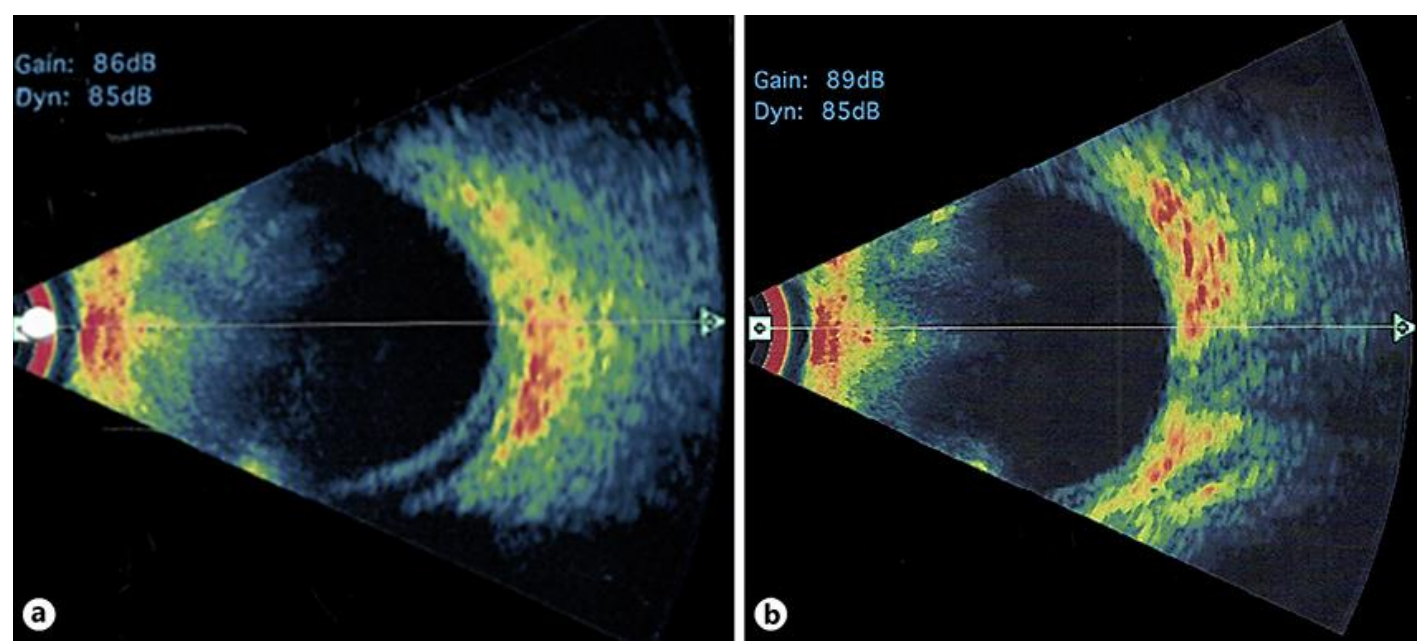

Fig. 3. B-scan findings. a, b B-scan findings of the right (a) and left (b) eye. B-scan of the right eye showed ciliochoroidal effusion while left eye B-scan was normal. 